\title{
A new busy signal-based MAC protocol supporting QoS for ad-hoc networks with hidden nodes
}

\author{
Katarzyna Kosek-Szott • Marek Natkaniec • \\ Andrzej R. Pach
}

Published online: 22 November 2012

(c) The Author(s) 2012. This article is published with open access at Springerlink.com

\begin{abstract}
This article presents three versions of a novel MAC protocol for IEEE 802.11 ad-hoc networks called Busy Signal-based Mechanism turned On (BusySiMOn) (This is an extended version of our conference paper: [15]). The key idea of the proposed solution is based on an intelligent two-step reservation procedure combined with the advantages of EDCA service differentiation. The former alleviates the hidden node problem while the latter ensures compatibility with the IEEE 802.11 standard. Simulation results obtained for saturated and non-saturated network conditions emphasize the advantages of the new protocol over the currently used four-way handshake mechanism in terms of fairness, throughput, and average frame delay.
\end{abstract}

Keywords EDCA - Hidden nodes - MAC protocol . QoS · Wireless communications

\section{Introduction}

The IEEE 802.11 standard is currently one of the most popular wireless access technologies. It allows quick and simple configuration of local broadband networks and

\footnotetext{
K. Kosek-Szott $(\square) \cdot$ M. Natkaniec $\cdot$ A. R. Pach

Department of Telecommunications, Faculty of Computer

Science, Electronics and Telecommunications, AGH University

of Science and Technology, al. A. Mickiewicza 30,

30-059 Krakow, Poland

e-mail: kosek@kt.agh.edu.pl

M. Natkaniec

e-mail: natkaniec@kt.agh.edu.pl

A. R. Pach

e-mail: pach@kt.agh.edu.pl
}

greatly facilitates Internet access. With the growth of the popularity of IEEE 802.11, the number of available services also increased and the need for Quality of Service (QoS) provisioning became apparent. As a remedy to this problem, the Enhanced Distributed Channel Access (EDCA) function of the IEEE 802.11 standard was proposed [8].

The IEEE 802.11 standard has a serious disadvantage resulting from the half-duplex nature of wireless devices. In each network with hidden nodes not only the overall throughput value may greatly decrease as shown in [34] but also EDCA service differentiation and throughput fairness among nodes may be strongly deteriorated [13].

A number of Medium Access Control (MAC) protocols attempting to address the problem of hidden nodes have been proposed in the literature. A detailed overview of such protocols is presented in Table 1. As can be noticed, the majority of protocols rely only on RTS/CTS or similar frame exchanges during the channel reservation process. All presented solutions can be divided into five major protocol types: contention-based, multi-channel, busy tonebased, energy-efficient and directional antenna-based. The most important advantages and disadvantages of each protocol type are presented in Table 2.

Among the available solutions, only the legacy four-way handshake mechanism has become broadly used and implemented in wireless devices. Currently it is the only mechanism recommended by the IEEE 802.11 standard to minimize the negative effects caused by hidden nodes. However, as it was shown in [13], the effectiveness of the four-way handshake is insufficient to provide appropriate service differentiation in EDCA-based ad-hoc networks.

In this article we describe three versions of Busy Signalbased Mechanism turned On (BusySiMOn), which combine a preliminary reservation of the wireless channel with 


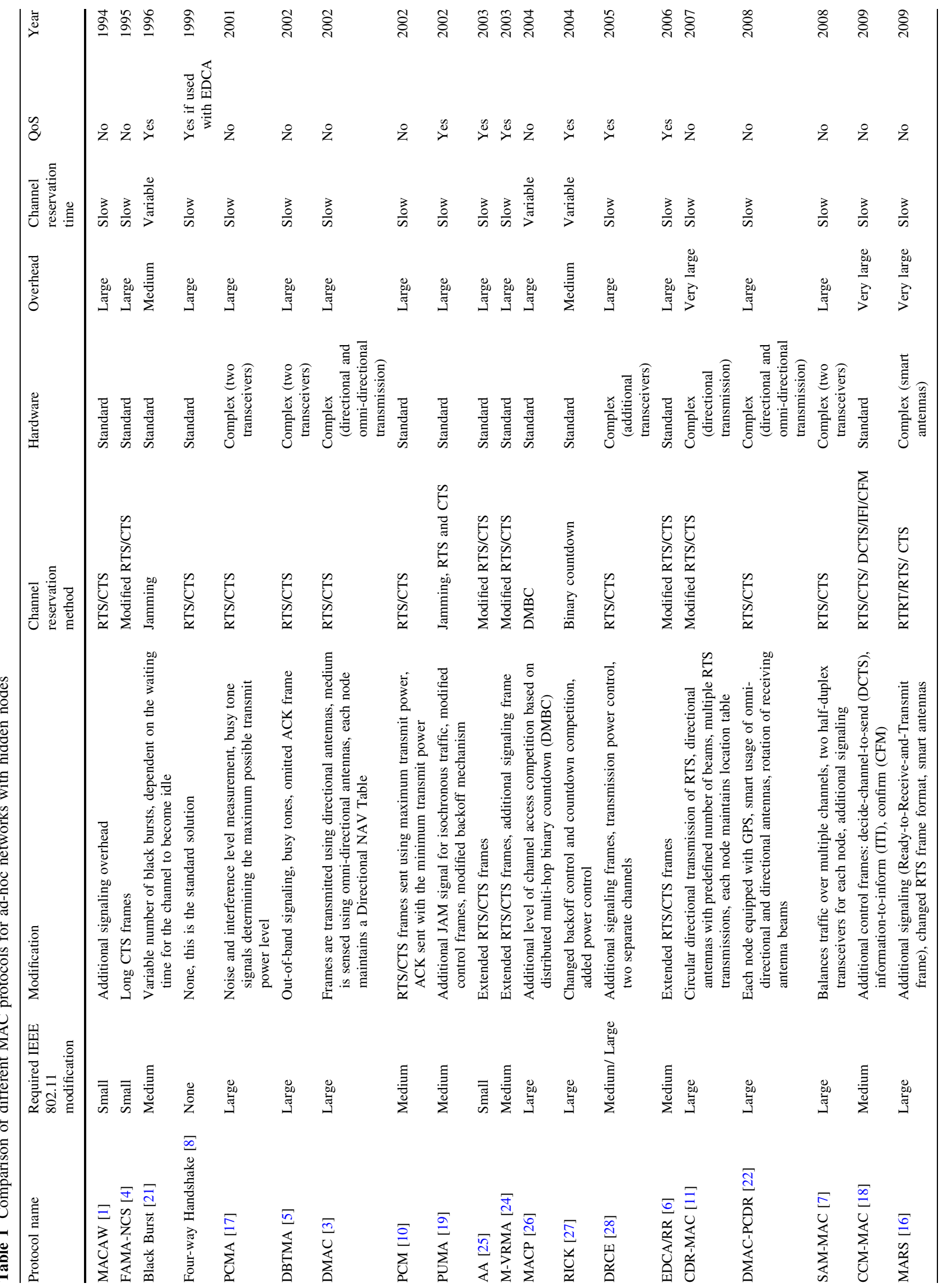




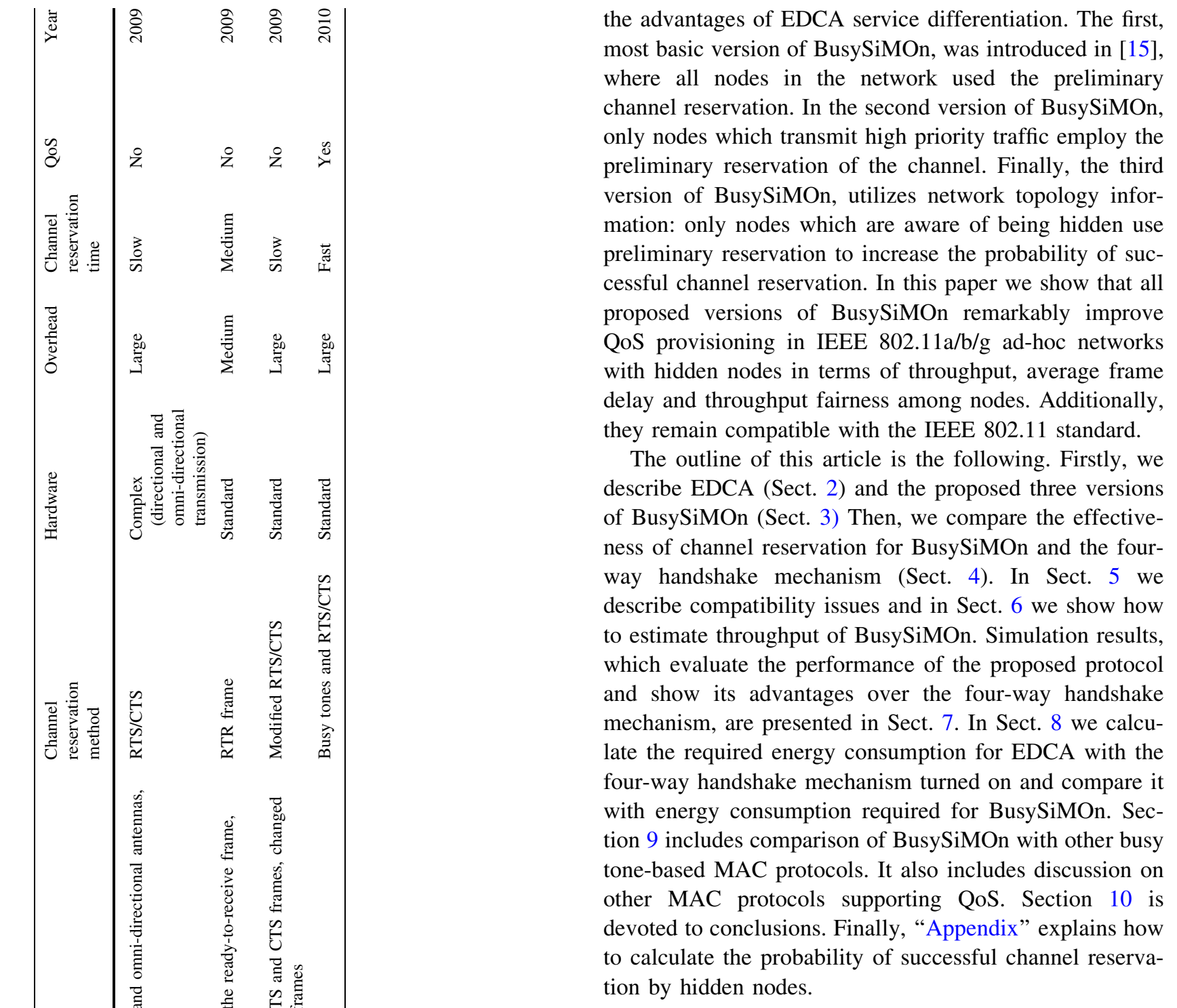

2 IEEE 802.11 EDCA

In networks with heterogeneous traffic, the QoS requirements of each service should be carefully taken into account. In particular, in the case of simultaneous transmissions of multimedia and data traffic the delay constraints of the multimedia service should be primarily met. To achieve this goal multimedia traffic should have priority over data traffic. Within wireless ad-hoc networks it is the EDCA function of the IEEE 802.11 standard which was designed to satisfy this requirement.

The EDCA function defines several QoS enhancements to the legacy IEEE 802.11 Distributed Coordination Function (DCF) which are based on the idea of Access Categories (ACs). Four ACs (priorities) are defined: Voice (VO), Video (VI), Best Effort (BE), and Background (BK). To provide traffic differentiation each $\mathrm{AC}$ has different values of the 
Table 2 Advantages and disadvantages of different MAC protocol types for ad-hoc networks with hidden nodes

\begin{tabular}{|c|c|c|}
\hline Protocol type & Advantages & Disadvantages \\
\hline $\begin{array}{l}\text { Pure contention-based (e.g., } \\
\text { MACA, four-way } \\
\text { handshake, EDCA/RR) }\end{array}$ & $\begin{array}{l}\text { Standard hardware. Interoperability with IEEE } 802.11 \\
\text { if the standard RTS and CTS frames are used }\end{array}$ & $\begin{array}{l}\text { Large signaling overhead. Slow channel reservation. } \\
\text { Often not suitable for delay sensitive traffic }\end{array}$ \\
\hline $\begin{array}{l}\text { Single channel busy tone- } \\
\text { based (e.g., PUMA, Black } \\
\text { Burst, BusySiMOn) }\end{array}$ & $\begin{array}{l}\text { Standard hardware. Easy recognition of busy tones. } \\
\text { Partial or full interoperability with IEEE } 802.11 \text {. } \\
\text { Quick channel reservation is possible }\end{array}$ & $\begin{array}{l}\text { Increased signaling overhead. Legacy nodes may be } \\
\text { assigned a lower priority }\end{array}$ \\
\hline $\begin{array}{l}\text { Multiple channel-based } \\
\text { (e.g., DBTMA, SAM- } \\
\text { MAC, CCM-MAC) }\end{array}$ & $\begin{array}{l}\text { Separation of data and control traffic to reduce } \\
\text { collisions. Possibility of load balancing and use of } \\
\text { busy tones. Simultaneous transmissions in the same } \\
\text { region without interference. Higher network } \\
\text { efficiency than legacy IEEE } 802.11\end{array}$ & $\begin{array}{l}\text { Assignment of separate channels must be done in real- } \\
\text { time. Nodes must sometimes be synchronized. } \\
\text { Hardware complexity because of additional channels } \\
\text { and transceivers. Channel gain of data and control } \\
\text { channels may be different. Nodes with a large number } \\
\text { of transceivers (e.g., one per channel) are expensive } \\
\text { while nodes equipped with a single transceiver are } \\
\text { inefficient. Difficult interoperability with existing } \\
\text { IEEE } 802.11 \text {. Large signaling overhead. Slow } \\
\text { channel reservation }\end{array}$ \\
\hline $\begin{array}{l}\text { Power-aware (e.g., DRCE, } \\
\text { PCM, SSPC) }\end{array}$ & $\begin{array}{l}\text { Decreased energy consumption. Can be combined with } \\
\text { busy tones or can take advantage of multiple channels }\end{array}$ & $\begin{array}{l}\text { Signal fading may degrade performance. Reducing the } \\
\text { power of ACK transmission may lead to increased } \\
\text { number of collisions due to decreased carrier sensing } \\
\text { range. Additional hardware complexity. Large } \\
\text { signaling overhead. Slow channel reservation }\end{array}$ \\
\hline $\begin{array}{l}\text { Directional antenna-based } \\
\text { (e.g., RDMAC, MARS, } \\
\text { MCDA, DMAC) }\end{array}$ & $\begin{array}{l}\text { Simultaneous data transmission and reception increases } \\
\text { spatial reuse. Minimized probability of collisions. } \\
\text { Higher network efficiency than IEEE } 802.11\end{array}$ & $\begin{array}{l}\text { New kinds of hidden nodes, higher directional } \\
\text { interference and deafness. Performance decreases } \\
\text { with node mobility. Additional hardware complexity. } \\
\text { In most cases large signaling overhead and slow } \\
\text { channel reservation. Performance strongly dependent } \\
\text { on network topology. Performance can be } \\
\text { deteriorated by the side-lobe problem }\end{array}$ \\
\hline
\end{tabular}

following medium access parameters: the contention window minimum ( $C W \min )$ and maximum $(C W \max )$ size, the arbitration inter-frame space number $(A I F S N)$, and the transmission opportunity limit (TXOPLimit).

The functions of the EDCA access parameters are as follows: $C W \min _{i}$ and $C W \max _{i}$ determine the number of Backoff $_{i}$ slots for the $i$-th $\mathrm{AC}$ :

$$
\begin{aligned}
& \text { Backoff }_{i}= \\
& =\operatorname{random}\left[0, \min \left(2^{k}\left(C W \min _{i}+1\right)-1, C W \max _{i}\right)\right]
\end{aligned}
$$

where $k$ is the number of collisions occurred to the currently transmitted frame. $A I F S N_{i}$ determines the minimum time interval before a frame transmission may begin $\left(A I F S_{i}\right)$ :

$$
A I F S_{i}=A I F S N_{i} \times T_{e}+S I F S,
$$

where $T_{e}$ is the duration of a single slot time and SIFS is the Short Inter-Frame Space. TXOPLimit allows for the consecutive transmission of several frames after gaining channel access, known as contention free bursting. This parameter is optional.

In the literature there are a number of articles which describe the advantages of EDCA traffic differentiation. Most of the studies, however, consider systems without hidden nodes. In [12] it has been proved that EDCA tends to cease to function in environments with hidden nodes. In particular, it has been shown that:

- unhidden nodes are generally favored over hidden nodes in the channel access, regardless of their access category,

- the four-way handshake mechanism does not completely eliminate unfairness in granting channel access,

- the higher the priority of traffic transmitted by hidden nodes the more collisions occur, even if the four-way handshake is used.

These observations were also confirmed in [13]. Therefore, it became obvious that a new MAC protocol is required to meet the strict demands of high priority traffic (VO and VI) and to improve fairness among nodes. It was also clear that the ideal solution should be compatible with both currently used mechanisms: EDCA and the four-way handshake. The proposed protocol, which satisfies all these requirements, is described in the next section.

\section{BusySiMOn}

The key idea of BusySiMOn is to minimize the probability of collisions of the signaling data within wireless networks with hidden nodes in comparison to the currently used four-way 


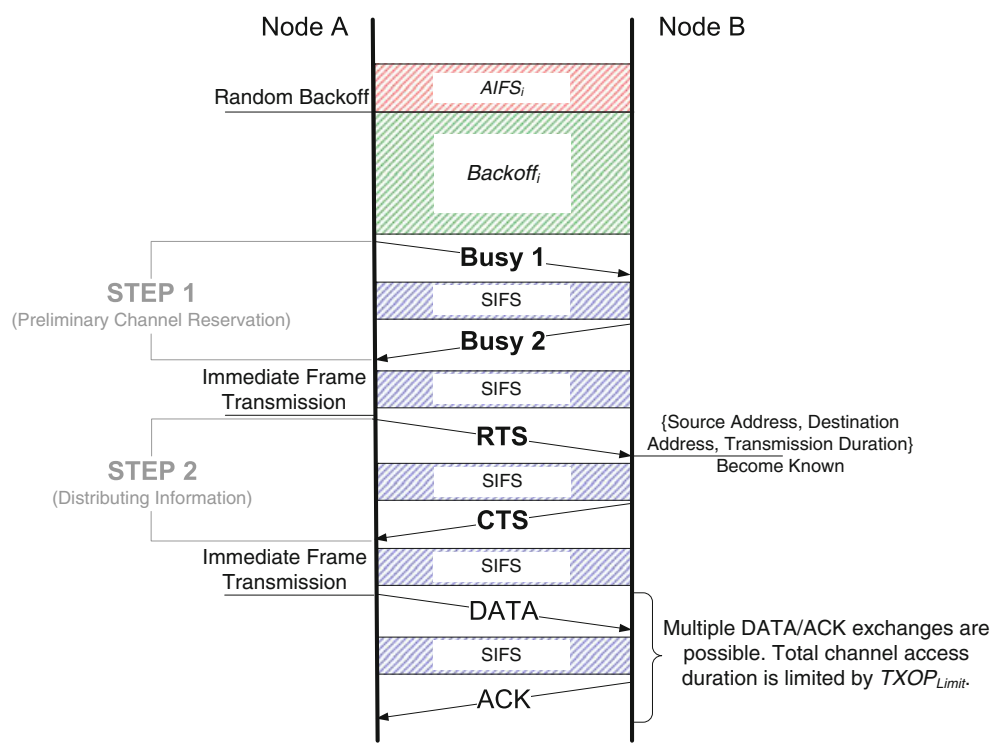

Fig. 1 Operation of BusySiMOn

handshake mechanism. Additionally, it was assumed that only slight modifications of the IEEE 802.11 standard are allowed in order to assure backward compatibility. To achieve these goals we propose a new channel reservation procedure consisting of the following two steps (cf., Fig. 1):

1. Preliminary reservation of the wireless channel using two busy tone signals (Busy 1 and Busy 2), i.e., pulses of energy of a predefined duration. Busy 1 is used to request channel reservation by a source node and Busy 2 is used to confirm this reservation by its neighboring nodes. Both signals are very short-Busy 1 has a length of one Slot Time Period (STP) and Busy 2 has a length of three STPs. Therefore, the preliminary channel reservation can be performed very quickly and the probability of collisions of signaling data can be meaningfully reduced in comparison to the fourway handshake mechanism.

2. Distributing information about the transmission duration as well as the source and destination node addresses with the use of the legacy RTS and CTS frames.

As illustrated in Fig. 1, the problem of traffic prioritization is resolved in BusySiMOn by the combination of the proposed reservation mechanism with the standard EDCA access parameters: AIFSN, CW, and TXOPLimit.

In this paper we propose the following three versions of BusySiMOn:

- BusySiMOn v1: all nodes within the network use Busy 1 and Busy 2 signals to preliminarily reserve the wireless channel [15].

- BusySiMOn v2: only nodes with high priority data (i.e., VO or VI) use the Busy 1/Busy 2 exchange to preliminarily reserve the wireless channel. Other nodes use the basic channel access (without RTS/CTS).

- BusySiMOn v3: only hidden nodes use the Busy 1/Busy 2 exchange to preliminarily reserve the wireless channel. Other nodes use the basic channel access. This version of BusySiMOn needs an additional hidden node detection scheme which is out of the scope of this work ${ }^{1}$. Therefore, in this paper ideal conditions are assumed, i.e., each hidden node is aware of being hidden.

\section{Effectiveness of channel reservation}

In the case of the legacy RTS/CTS-based channel reservation three types of collisions may happen-collisions of RTS with either another RTS, CTS or DATA. They are common even for the simplest line topology depicted in Fig. 2. In the first scenario two RTS frames sent by the hidden nodes collide with each other. After the collision is detected they have to be retransmitted after a random Backoff time. The number of possible retransmissions is limited to the Short Retry Limit defined by the IEEE 802.11 standard. It is worth noting that, due to the low sending rate of RTS frames ${ }^{2}$, hidden nodes do not have to simultaneously start their RTS transmissions to cause a collision. In the second scenario, node N1 succeeds in reserving the wireless channel with the use of the RTS/CTS exchange. At the same time, however, the RTS frame sent by N3

\footnotetext{
${ }_{1}$ To assure rapid detection of hidden nodes the Network layer of the OSI model can be used because network topology information can be obtained from routing tables. Exemplary hidden node detection schemes are described in [29-32].

${ }^{2}$ E.g., the IEEE $802.11 \mathrm{~b}$ standard recommends $1 \mathrm{Mb} / \mathrm{s}$ as the transmission rate of the PLCP overhead.
} 


\section{SCENARIO 1}

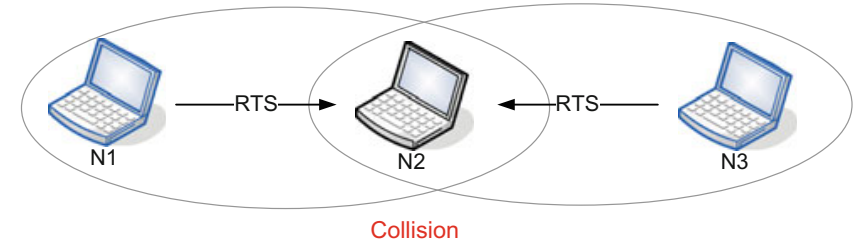

RTS is much longer than Busy 1: the probability of collision is high

SCENARIO 2
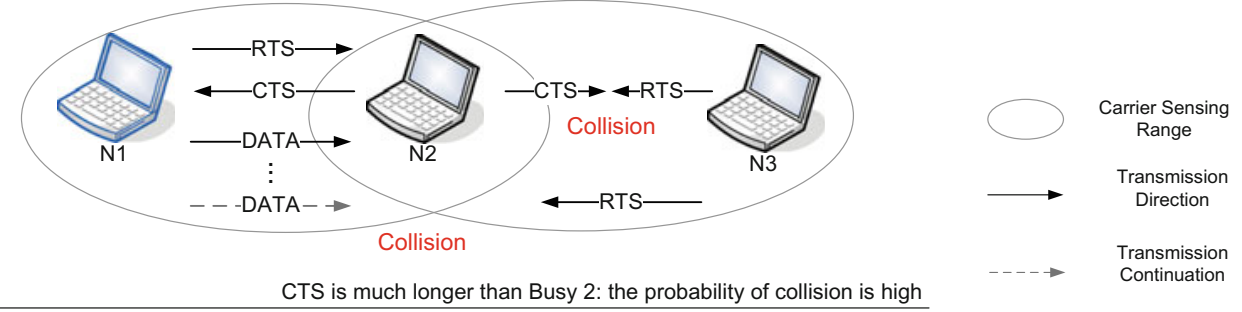

Fig. 2 Types of signaling collisions for the four-way handshake

collides with the CTS frame sent by N2. Obviously, after a random Backoff time, N3 will attempt to resend its RTS frame. If the Backoff value will be small enough, the resent RTS frame will collide with the DATA frame currently being transmitted by N1. As a result, N1 will have to resend its DATA frame.

For a given Backoff stage, with the use of simple probability analysis (which is explained in "Appendix"), we can compute the lower bound of the probability of a successful channel reservation by either of the two hidden nodes $\left(p_{s}^{H}\right)$ in the first scenario:

$p_{s}^{H, R T S}=\left\{\begin{array}{cl}0, & \text { if } \alpha<0, \\ \frac{(\alpha+1)(\alpha+2)}{(C W+1)^{2}}, & \text { if } \alpha \geq 0,\end{array}\right.$

where $\alpha=C W-T_{R T S}, C W$ is the current contention window size (in STPs), and $T_{R T S}$ is the number of STPs required to transmit the RTS frame (together with its PLCP header and preamble) $\left(T^{R T S}\right)$ and wait a SIFS period ${ }^{3}$.

If Busy 1 was used to reserve the wireless channel the probability $p_{s}^{H}$ would be the following:

$p_{s}^{H, \text { Busy } 1}=\left\{\begin{array}{cl}0, & \text { if } \beta<0 \\ \frac{(\beta+1)(\beta+2)}{(C W+1)^{2}}, & \text { if } \beta \geq 0\end{array}\right.$

where $\beta=C W-T_{\text {Busy } 1}$ and $T_{\text {Busy } 1}$ is the number of STPs required to transmit the Busy 1 signal $\left(T^{B u s y 1}\right)$ and wait a SIFS period ${ }^{4}$.

The comparison of $p_{s}^{H, R T S}$ with $p_{s}^{H, B u s y}{ }^{1}$ for different PHYs and different $C W$ values is given in Table 3 . The probability $p_{s}^{H, B u s y}{ }^{1}$ is always greater than $p_{s}^{H, R T S}$ because

\footnotetext{
${ }^{3} T_{R T S}=\left\lceil T^{R T S}+S I F S\right\rceil$

${ }^{4} T_{B u s y 1}=\left\lceil T^{B u s y 1}+S I F S\right\rceil$
}

the proposed solution maximizes the probability of successful reservations of the wireless channel for hidden nodes by minimizing the probability of collisions of signaling data.

To assess the effectiveness of the four-way handshake we compare different $C W$ values (Table 3) with the standard values of $C W \min$ and $C W \max$ of different ACs [8]. By analyzing $p_{s}^{H, R T S}$ it can be deduced that especially for hidden nodes with VO priority flows the probability of successful channel reservation is very low for each PHY when the four-way handshake is used. For BusySiMOn the probability $p_{s}^{H, B u s y 1}$ for VO priority flows is much higher.

\section{Compatibility with EDCA}

The BusySiMOn protocols are compatible with EDCA because they do not change the values of the channel access parameters defined by the IEEE 802.11 standard. Furthermore, because the RTS/CTS/DATA/ACK exchange is part of the proposed solutions, each node implementing the preliminary BusySiMOn channel reservation is able to respond to legacy IEEE 802.11 nodes. Additionally, if a node using Busy 1/Busy 2 exchange wants to communicate with a legacy node it must have at least one other $\mathrm{Bu}-$ sySiMOn neighbor. For example, assume that in Fig. 3, nodes $\mathrm{A}$ and $\mathrm{B}$ implement BusySiMOn while node $\mathrm{C}$ is a legacy node. After node B broadcasts Busy 1 to all nodes within its range, node $A$ sends Busy 2 in response. This allows node $\mathrm{B}$ to communicate with node $\mathrm{C}$ with the use of the traditional RTS/CTS/ DATA/ACK exchange.

A problem occurs if a BusySiMOn node does not have any BusySiMOn neighbors. To overcome this obstacle and 
Table 3 Lower bound of the probability of successful transmission by either of the hidden nodes in the first scenario in Fig. 2 for different PHYs

\begin{tabular}{|c|c|c|c|c|c|c|c|c|c|c|}
\hline IEEE amendment & PHY & $\begin{array}{l}\text { PLCP header } \\
\text { and preamble } \\
(\mu \mathrm{s})\end{array}$ & $\begin{array}{l}\text { Slot time } \\
(\mu \mathrm{s})\end{array}$ & $\begin{array}{l}\text { SIFS } \\
(\mu \mathrm{s})\end{array}$ & $\begin{array}{l}\text { Tx Rate } \\
(\mathrm{Mb} / \mathrm{s})\end{array}$ & $\begin{array}{l}C W \\
\text { (STP) }\end{array}$ & $\begin{array}{l}T_{R T S} \\
\text { (STP) }\end{array}$ & $p_{s}^{H, R T S}$ & $\begin{array}{l}T_{\text {Busy } 1} \\
\text { (STP) }\end{array}$ & $p_{s}^{H, \text { Busy } 1}$ \\
\hline \multirow[t]{5}{*}{$802.11 b$} & HR/DSSS & 192 & 20 & 10 & $1-11$ & 7 & $19-11$ & 0.00 & 2 & 0.66 \\
\hline & & & & & & 15 & & $0.00-0.12$ & & 0.82 \\
\hline & & & & & & 31 & & $0.18-0.45$ & & 0.91 \\
\hline & & & & & & 63 & & $0.51-0.70$ & & 0.95 \\
\hline & & & & & & 1,023 & & $0.96-0.98$ & & 1.00 \\
\hline \multirow[t]{5}{*}{$802.11 \mathrm{~g}$} & OFDM & 20 & 9 & 10 & $6-54$ & 7 & $7-5$ & $0.03-0.19$ & 3 & 0.47 \\
\hline & & & & & & 15 & & $0.35-0.52$ & & 0.71 \\
\hline & & & & & & 31 & & $0.63-0.74$ & & 0.85 \\
\hline & & & & & & 63 & & $0.81-0.86$ & & 0.92 \\
\hline & & & & & & 1,023 & & 0.99 & & 1.00 \\
\hline \multirow[t]{15}{*}{$802.11 \mathrm{a}$} & OFDM & 20 & 9 & 16 & $6-54$ & 7 & $7-5$ & $0.03-0.19$ & 3 & 0.47 \\
\hline & & & & & & 15 & & $0.35-0.52$ & & 0.71 \\
\hline & & & & & & 31 & & $0.63-0.74$ & & 0.85 \\
\hline & & & & & & 63 & & $0.81-0.86$ & & 0.92 \\
\hline & & & & & & 1,023 & & 0.99 & & 1.00 \\
\hline & & 40 & 13 & 32 & $6-54$ & 7 & $8-6$ & $0.00-0.09$ & 4 & 0.31 \\
\hline & & & & & & 15 & & $0.28-0.43$ & & 0.61 \\
\hline & & & & & & 31 & & $0.59-0.69$ & & 0.79 \\
\hline & & & & & & 63 & & $0.78-0.84$ & & 0.89 \\
\hline & & & & & & 1,023 & & 0.99 & & 0.99 \\
\hline & & 80 & 21 & 64 & $6-54$ & 7 & $9-7$ & $0.00-0.03$ & 5 & 0.19 \\
\hline & & & & & & 15 & & $0.22-0.35$ & & 0.52 \\
\hline & & & & & & 31 & & $0.54-0.63$ & & 0.74 \\
\hline & & & & & & 63 & & $0.75-0.81$ & & 0.86 \\
\hline & & & & & & 1,023 & & $0.98-0.99$ & & 0.99 \\
\hline
\end{tabular}

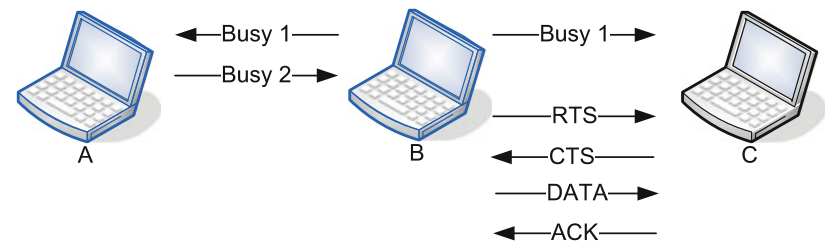

Fig. 3 Compatibility with legacy nodes

assure full compatibility with the IEEE 802.11 standard, the BusySiMOn protocol is extended in the following way. If a node implementing BusySiMOn does not receive a reply to $m$ Busy 1 tones it assumes that all other nodes use the legacy IEEE 802.11. It then reverts to the traditional four-way handshake exchange. Additionally, a node implementing BusySiMOn can overhear Busy 1 signals from its neighboring nodes. Therefore, it becomes aware if a BusySiMOn-capable node appears within its range. After such a node is detected, it automatically falls back to normal operation. Furthermore, each node implementing BusySiMOn periodically transmits the Busy 1 signal with a probability proportional to the time it did not hear any BusySiMOn-like transmission. The probability of transmitting the Busy 1 signal is a protocol parameter that can be adjusted, e.g., depending on the network topology.

\section{Throughput estimation}

In this section we briefly present saturation throughput models of EDCA, the four-way handshake, and BusySiMOn. This allows to estimate the signaling overhead and compare it with simulation results in Sect. 7.1.2. Good agreement of theoretical and simulation results validates the correct implementation of BusySiMOn in the ns-2 simulator

The analysis presented in this section is based on the EDCA throughput model that we proposed in [14]. For EDCA, the throughput of the $i$-th AC $\left(S_{i}\right)$ is equal to the average duration of a successful transmission of a frame in this particular AC divided by the average duration of a 
contention slot $\left(T^{C S}\right)$, in which the frame competes for medium access with all other frames:

$S_{i}=\frac{p_{i}^{S} T^{D A T A}}{T^{C S}}$,

where $p_{i}^{S}$ is the probability of a successful transmission for a given $\mathrm{AC}$ and $T^{D A T A}$ is the average time spent on transmitting a frame.

A single transmission is successful if only one node transmits its data in a given slot time. Therefore, if $\tau_{i}$ is the probability of a transmission attempt in a generic slot time for the $i$-th AC, then

$p_{i}^{S}=n_{i} \tau_{i}\left(1-\tau_{i}\right)^{n_{i}-1} \prod_{\substack{j=0 \\ j \neq i}}^{N_{c}-1}\left(1-\tau_{j}\right)^{n_{j}}$,

where $n_{i}$ is the number of nodes in the $i$-th $\mathrm{AC}$ and $N_{c}$ is the number of ACs.

If $T_{e}$ is the slot time, $T^{S}$ the duration of a successful transmission, $T^{C}$ the duration of a collision, $p^{B}$ the probability of a busy medium, and $\left(1-p^{B}\right)$ the probability of a free channel, we can rewrite $T^{C S}$ as $\left(1-p^{B}\right) T_{e}+\sum_{i=0}^{N_{c}-1}$ $p_{i}^{S} T^{S}+\left(p^{B}-\sum_{i=0}^{N_{c}-1} p_{i}^{S}\right) T^{C}$. Therefore, we get:

$S_{i}=\frac{p_{i}^{S} T^{D A T A}}{\left(1-p^{B}\right) T_{e}+\sum_{i=0}^{N_{c}-1} p_{i}^{S} T^{S}+\left(p^{B}-\sum_{i=0}^{N_{c}-1} p_{i}^{S}\right) T^{C}}$,

where $p^{B}=1-\prod_{j=0}^{N_{c}-1}\left(1-\tau_{j}\right)^{n_{j}}$.

Time intervals $T^{S}$ and $T^{C}$ depend on the access method used. In the case of the basic access method they are as follows:

$T^{S}=A I F S_{i}+T^{D A T A}+S I F S+T^{A C K}+2 \delta$,

$T^{C}=T^{D A T A}+\delta+A C K^{\text {Timeout }}+A I F S_{i}$,

where $\delta$ denotes the propagation delay and $A C K^{\text {Timeout }}$ equals EIFS - DIFS.

In the case of the four-way handshake mechanism $T^{S}$ and $T^{C}$ are represented by the following equations:

$$
\begin{aligned}
T^{S} & =T^{R T S}+T^{C T S}+A I F S_{i}+T^{D A T A}+3 \times \text { SIFS }+ \\
& +T^{A C K}+4 \delta, \\
T^{C} & =T^{R T S}+C T S^{\text {Timeout }}+A I F S_{i},
\end{aligned}
$$

where $C T S^{\text {Timeout }}=E I F S-D I F S . T^{R T S}$ and $T^{C T S}$ is the time required to send the RTS and CTS frames, respectively.

In the case of BusySiMOn v1 (which exhibits the largest overhead) $T^{S}$ and $T^{C}$ are represented by the following equations:

$$
\begin{aligned}
T^{S} & =T^{\text {Busy1 }}+T^{\text {Busy2 }}+T^{R T S}+T^{C T S}+\text { AIFS }_{i}+ \\
& +T^{\text {DATA }}+5 \times \operatorname{SIFS}+T^{A C K}+4 \delta \\
T^{C} & =T^{\text {Busy1 }}+T^{\text {Busy } 2}+2 \times S I F S+T^{R T S}+ \\
& + \text { CTS }^{\text {Timeout }}+\text { AIFS }_{i},
\end{aligned}
$$

where $T^{\text {Busy } 1}$ and $T^{\text {Busy } 2}$ is the time required to send the Busy 1 and Busy 2 signals, respectively. The remaining unknown variables $\left(\tau_{i}, p_{i}^{S}, p_{i}^{B}\right)$ can be calculated using the model presented in [14].

\section{Simulation study}

The BusySiMOn protocols were implemented in the ns2.28 simulator, which was modified to allow the coexistence of three types of nodes: those which implement the Busy 1/Busy 2 exchange, those which use basic channel access method, and those which employ the four-way handshake mechanism.

In the simulations we assumed that the wireless channel introduced no errors, i.e., frame losses occurred only because of collisions. IEEE $802.11 \mathrm{~b}$ was chosen as the PHY layer, although the BusySiMOn protocols can be applied to any other 802.11 PHY. The general conclusions presented in this section remain the same regardless of the chosen PHY. The EDCA parameters were set as defined by the IEEE 802.11 standard [8]. TXOPLimit was set to zero to avoid contention free bursting. Finally, in order to simulate the topologies with hidden nodes the Carrier Sensing Range (CSR) was decreased (from 550 to $263 \mathrm{~m}$ ) so that every hidden node was out of the range of other hidden nodes. There were no exposed nodes. Networks meeting these requirements are presented in Fig. 4. They were used during simulations. Each simulation was repeated until for $95 \%$ confidence intervals the error was smaller than $2 \%$.

Four different simulation scenarios are described in this section:

- Scenario 1: One AC per node, no hidden nodes, simple network topology. Goal: overhead study under saturation.

- Scenario 2: One AC per node, multiple hidden nodes, four network topologies. Goal :performance study under saturation.

- Scenario 3: One AC per node, multiple hidden nodes, complex network topology. Goal: performance study under non-saturation.

- Scenario 4: Four ACs per node, multiple hidden nodes, complex network topology, saturation. Goal: study of the impact of virtual collisions.

These scenarios allowed to answer the following questions regarding BusySiMOn: how large is the signaling overhead, what is the performance under saturation and nonsaturation, and what is the impact of virtual collisions on the protocol performance? 


\subsection{One AC per node}

\subsubsection{Evaluation criteria}

The evaluation was done in terms of throughput and fairness obtained for different values of per-flow offered load. The per-flow offered load is the total number of bits generated by a single node for a single flow per time unit (second). Throughput is defined as the ratio of the number of correctly received bits per time unit. In the results presented in this section only the overall network throughput is considered. Fairness is measured with Jain's fairness index [9]:

Fairness $=\frac{\left(\sum x_{i}\right)^{2}}{n \sum x_{i}^{2}}$

where $x_{i}$ is the average throughput of the $i$-th node for a particular AC and $n$ is the number of nodes transmitting data with this AC.

If not defined differently the following four configurations were considered for networks presented in this section:

- Configuration 1: all nodes transmit VO traffic. In this case the competition among all nodes is the most severe due to low values of the EDCA medium access parameters.

- Configuration 2: all nodes transmit BK traffic. In this case the competition among nodes is the weakest due to high values of the EDCA medium access parameters.

- Configuration 3: node N0 transmits BK traffic, all other nodes transmit VO traffic. In this case the competition among hidden nodes is very severe due to low values of the EDCA medium access parameters.

- Configuration 4: node N0 transmits VO traffic, all other nodes transmit BK traffic. In this case the competition

(a)
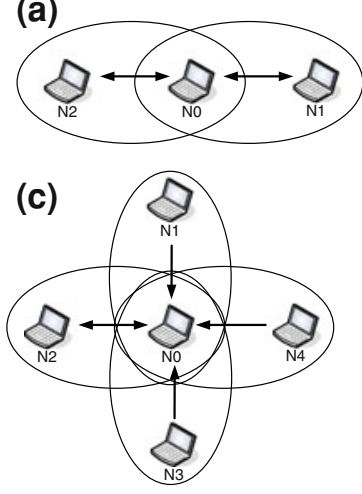

(b)

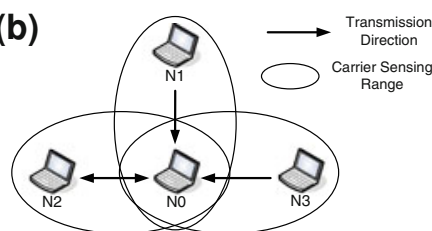

(d)

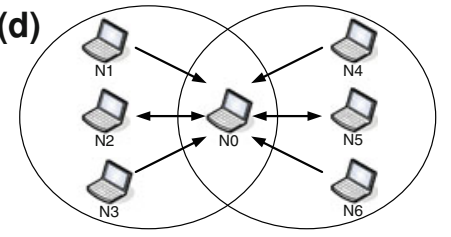

Fig. 4 Networks with hidden nodes: a three-node star topology network b four-node star topology network $\mathbf{c}$ five-node star topology network d complex star topology network among hidden nodes is weak due to high values of the EDCA medium access parameters.

Additionally, the most complex network (Fig. 4d) was evaluated in terms of the maximum frame delay defined as the maximum time difference between frame generation at the source node and its successful reception at the destination node. This was computed separately for each simulated AC.

From the list of available MAC protocols for networks with hidden nodes (Table 1) the four-way handshake was chosen for the presented comparison. This is because, as it was previously mentioned, the four-way handshake mechanism is the only solution recommended by the IEEE 802.11 standard to be used in environments with hidden nodes and it is the only solution implemented in current wireless drivers. Additional discussion on the differences between BusySiMOn and other QoS-aware MAC protocols is provided in Sect. 9.

\subsubsection{Scenario 1: overhead study}

In order to compare the impact of the overhead introduced by BusySiMOn v1 (i.e., the additional Busy 1 and Busy 2 signals) and the four-way handshake mechanism, a twonode network was investigated. Both simulation results (obtained from the ns-2 simulator) and mathematical results (calculated with the use of the model described in Sect. 6) are gathered in Table 4. Good agreement of simulation and theoretical results validates the correct implementation of BusySiMOn in ns-2.

The impact of the overhead $(\mathrm{OH})$ on the network performance was calculated using the following equations:

$O H_{4 W H}=\frac{\left|O N T_{E D C A}-O N T_{4 W H}\right|}{O N T_{E D C A}} \times 100 \%$

and

$O H_{B S}=\frac{\left|O N T_{E D C A}-O N T_{B S}\right|}{O N T_{E D C A}} \times 100 \%$,

where $O N T_{E D C A}, O N T_{4 W H}$, and $O N T_{B S}$ stand for the overall network throughput for EDCA, the four-way handshake, and BusySiMOn v1, respectively.

Table 4 Overhead study for two-node network under saturation

\begin{tabular}{lll}
\hline Configuration & $\mathrm{OH}_{4 W H}($ Simulation $)$ & $\mathrm{OH}_{4 W H}($ Model $)$ \\
1: N0, N1 = VO & $31 \%$ & $30 \%$ \\
2: N0, N1 = BK & $29 \%$ & $29 \%$ \\
3: N0 = VO, N1 = BK & $31 \%$ & $32 \%$ \\
Configuration & $\mathrm{OH}_{B S}$ (Simulation) & $O H_{B S}$ (Model) \\
1: N0, N1 = VO & $30 \%$ & $32 \%$ \\
2: N0, N1 = BK & $30 \%$ & $31 \%$ \\
3: N0 $=\mathrm{VO}, \mathrm{N} 1=\mathrm{BK}$ & $32 \%$ & $34 \%$ \\
\hline
\end{tabular}


Table 5 Simulation results

\begin{tabular}{|c|c|c|c|c|c|c|c|c|c|c|c|c|}
\hline $\mathrm{C}$ & $\begin{array}{l}\text { ONT } \\
\text { (EDCA) }\end{array}$ & $\begin{array}{l}\text { F } \\
\text { (EDCA) }\end{array}$ & $\begin{array}{l}\text { ONT } \\
(4 \mathrm{WH})\end{array}$ & $\begin{array}{l}\text { F } \\
(4 \mathrm{WH})\end{array}$ & $\begin{array}{l}\text { ONT (w/o } \\
\text { HN) }\end{array}$ & $\begin{array}{l}\text { F (w/o } \\
\mathrm{HN})\end{array}$ & $\begin{array}{l}\text { ONT } \\
\text { (BS1) }\end{array}$ & $\begin{array}{l}\text { F } \\
\text { (BS1) }\end{array}$ & $\begin{array}{l}\text { ONT } \\
\text { (BS2) }\end{array}$ & $\begin{array}{l}\text { F } \\
\text { (BS2) }\end{array}$ & $\begin{array}{l}\text { ONT } \\
\text { (BS3) }\end{array}$ & $\begin{array}{l}\text { F } \\
\text { (BS3) }\end{array}$ \\
\hline \multicolumn{13}{|c|}{ Three-node network (Fig. 4a) } \\
\hline 1 & 90.29 & 0.33 & 298.95 & 0.50 & 594.21 & 1 & 434.54 & 0.89 & 434.54 & 0.89 & 499.71 & 0.93 \\
\hline 2 & 538.35 & 0.75 & 417.44 & 0.81 & 600.09 & 1 & 422.60 & 0.98 & 538.35 & 0.75 & 479.78 & 0.98 \\
\hline 3 & 3.90 & N/A & 109.27 & N/A & 640.64 & N/A & 413.13 & N/A & 415.21 & N/A & 415.21 & N/A \\
\hline 4 & 706.47 & N/A & 474.66 & N/A & 705.74 & N/A & 466.70 & N/A & 465.75 & N/A & 707.19 & N/A \\
\hline \multicolumn{13}{|c|}{ Four-node network (Fig. 4b) } \\
\hline 1 & 15.39 & 0.25 & 141.04 & 0.54 & 562.92 & 1 & 418.10 & 0.82 & 418.10 & 0.82 & 468.10 & 0.88 \\
\hline 2 & 445.28 & 0.68 & 402.49 & 0.61 & 597.55 & 1 & 421.10 & 0.94 & 445.28 & 0.68 & 471.08 & 0.95 \\
\hline 3 & 0.5 & N/A & 62.29 & N/A & 594.60 & N/A & 388.84 & N/A & 389.17 & N/A & 398.17 & N/A \\
\hline 4 & 450.67 & N/A & 408.66 & N/A & 632.29 & N/A & 437.91 & N/A & 393.25 & N/A & 510.33 & N/A \\
\hline \multicolumn{13}{|c|}{ Five-node network (Fig. 4c) } \\
\hline 1 & 55.27 & 0.25 & 31.38 & 0.61 & 525.57 & 1 & 402.54 & 0.78 & 402.54 & 0.78 & 438.60 & 0.83 \\
\hline 2 & 398.22 & 0.51 & 391.34 & 0.50 & 588.79 & 1 & 419.61 & 0.89 & 398.22 & 0.51 & 464.77 & 0.90 \\
\hline 3 & 2.44 & N/A & 77.36 & N/A & 553.04 & N/A & 361.38 & N/A & 376.51 & N/A & 376.51 & N/A \\
\hline 4 & 401.05 & N/A & 396.71 & N/A & 627.07 & N/A & 437.31 & N/A & 356.92 & N/A & 508.90 & N/A \\
\hline \multicolumn{13}{|c|}{ Complex star topology (Fig. 4d) } \\
\hline 1 & 9.18 & 0.25 & 224.17 & 0.39 & 536.88 & 1 & 349.29 & 0.88 & 349.29 & 0.88 & 421.32 & 0.74 \\
\hline 2 & 427.76 & 0.43 & 370.43 & 0.55 & 609.38 & 1 & 406.02 & 0.93 & 427.76 & 0.43 & 450.96 & 0.86 \\
\hline 3 & 0.00 & N/A & 94.43 & N/A & 575.00 & N/A & 368.32 & N/A & 373.00 & N/A & 373.00 & N/A \\
\hline 4 & 647.52 & N/A & 473.80 & N/A & 681.19 & N/A & 462.97 & N/A & 462.54 & N/A & 663.45 & N/A \\
\hline
\end{tabular}

$C$ configuration, $O N T$ overall network throughput $[\mathrm{KB} / \mathrm{s}], F$ fairness, $4 \mathrm{WH}$ EDCA with RTS/CTS, w/o HN EDCA without hidden nodes, $B S 1$ BusySiMOn v1, BS2 BusySiMOn v2, BS3 BusySiMOn v3

In general the overhead introduced by the BusySiMOn signaling impacts the analyzed network performance more than the overhead introduced by the four-way handshake mechanism. Obviously, such performance was expected because BusySiMOn introduces additional Busy 1 and Busy 2 signals to the traditional four-way handshake exchange. However, the overhead is only slightly larger than for the four-way handshake and is offset by the improved performance of BusySiMOn, as described in the following subsections.

\subsubsection{Scenario 2: performance study under saturation}

When analyzing the performance of the BusySiMOn protocols under saturation the following set of outcomes was expected:

1. the individual throughput values of the nodes should be the highest,

2. the overall network throughput should be similar to that of EDCA without hidden nodes and without RTS/CTS,

3. the protocol should perform the best when VO is the dominant traffic in the network (Configurations 1 and 3 ), slightly worse performance for BK traffic can be accepted (Configurations 2 and 4),

4. the Jain's fairness index should be near to one,
5. the above outcomes should be valid regardless of the configuration.

7.1.3.1 Results The performance of the new protocols was evaluated in four exemplary wireless ad-hoc networks with hidden nodes (Fig. 4). In each network node N0 was the only unhidden node. Other nodes, which belong to different collision domains (defined in this article as carrier sensing ranges), were hidden from each other. In our simulations we do not consider networks with exposed nodes because BusySiMOn was designed to minimize only the impact of hidden nodes.

The simulation results, gathered for the four configurations defined in Sect. 7.1.1, are presented in Table 5. These are the results from simulating five different MAC protocols (i.e., EDCA, EDCA with RTS/CTS, BusySiMOn v1, BusySiMOn v2, and BusySiMOn v3) in four different networks (Fig. 4). Furthermore, the tables contain results obtained for networks without hidden nodes. ${ }^{5}$

Based on the gathered results a comparison of the protocol efficiency was performed (Table 6). The comparison

\footnotetext{
5 These networks were formed by extending the CSR of the nodes for each of the analyzed networks so that all nodes were within the range of each other.
} 
Table 6 Comparison of protocol efficiency

\begin{tabular}{|c|c|c|c|}
\hline Configuration & Overall network throughput & Fairness & Similarity \\
\hline \multicolumn{4}{|c|}{ Three-node network (Fig. 4a) } \\
\hline 1 & EDCA $<4 \mathrm{WH}<\mathrm{BS} 1, \mathrm{BS} 2<\mathrm{BS} 3$ & $\mathrm{EDCA}<4 \mathrm{WH}<\mathrm{BS} 1, \mathrm{BS} 2<\mathrm{BS} 3$ & BS3: $84 \%$ \\
\hline 2 & $4 \mathrm{WH}<\mathrm{BS} 1<\mathrm{BS} 3<\mathrm{BS} 2, \mathrm{EDCA}$ & EDCA, BS $2<4 \mathrm{WH}<\mathrm{BS} 1, \mathrm{BS} 3$ & BS3: $80 \%$ \\
\hline 3 & $\mathrm{EDCA}<4 \mathrm{WH}<\mathrm{BS} 1, \mathrm{BS} 2, \mathrm{BS} 3$ & N/A & BS3: $65 \%$ \\
\hline 4 & $\mathrm{BS} 1, \mathrm{BS} 2<4 \mathrm{WH}<\mathrm{EDCA}, \mathrm{BS} 3$ & N/A & BS3: $100 \%$ \\
\hline \multicolumn{4}{|c|}{ Four-node network (Fig. 4b) } \\
\hline 1 & EDCA $<4 \mathrm{WH}<\mathrm{BS} 1, \mathrm{BS} 2<\mathrm{BS} 3$ & $\mathrm{EDCA}<4 \mathrm{WH}<\mathrm{BS} 1, \mathrm{BS} 2<\mathrm{BS} 3$ & BS3: $83 \%$ \\
\hline 2 & $4 \mathrm{WH}<\mathrm{BS} 1<\mathrm{EDCA}, \mathrm{BS} 2<\mathrm{BS} 3$ & $4 \mathrm{WH}<\mathrm{EDCA}, \mathrm{BS} 2<\mathrm{BS} 1<\mathrm{BS} 3$ & BS3: $79 \%$ \\
\hline 3 & $\mathrm{EDCA}<4 \mathrm{WH}<\mathrm{BS} 1, \mathrm{BS} 2, \mathrm{BS} 3$ & N/A & BS3: $65 \%$ \\
\hline 4 & $\mathrm{BS} 2<4 \mathrm{WH}<\mathrm{BS} 1<\mathrm{EDCA}<\mathrm{BS} 3$ & N/A & BS3: $81 \%$ \\
\hline \multicolumn{4}{|c|}{ Five-node network (Fig. 4c) } \\
\hline 1 & $4 \mathrm{WH}<\mathrm{EDCA}<\mathrm{BS} 1, \mathrm{BS} 2<\mathrm{BS} 3$ & $\mathrm{EDCA}<4 \mathrm{WH}<\mathrm{BS} 1, \mathrm{BS} 2<\mathrm{BS} 3$ & BS3: $83 \%$ \\
\hline 2 & $4 \mathrm{WH}<\mathrm{EDCA}, \mathrm{BS} 2<\mathrm{BS} 1<\mathrm{BS} 3$ & $4 \mathrm{WH}<\mathrm{EDCA}, \mathrm{BS} 2<\mathrm{BS} 1<\mathrm{BS} 3$ & BS3: $79 \%$ \\
\hline 3 & $\mathrm{EDCA}<4 \mathrm{WH}<\mathrm{BS} 1<\mathrm{BS} 2, \mathrm{BS} 3$ & N/A & BS3: $68 \%$ \\
\hline 4 & $\mathrm{BS} 2<4 \mathrm{WH}<\mathrm{EDCA}<\mathrm{BS} 1<\mathrm{BS} 3$ & N/A & BS3: $81 \%$ \\
\hline \multicolumn{4}{|c|}{ Complex star topology (Fig. 4d) } \\
\hline 1 & EDCA $<4 \mathrm{WH}<\mathrm{BS} 1, \mathrm{BS} 2<\mathrm{BS} 3$ & EDCA $<4 \mathrm{WH}<\mathrm{BS} 3<\mathrm{BS} 1, \mathrm{BS} 2$ & BS3: $78 \%$ \\
\hline 2 & $4 \mathrm{WH}<\mathrm{BS} 1<\mathrm{EDCA}, \mathrm{BS} 2<\mathrm{BS} 3$ & $\mathrm{EDCA}, \mathrm{BS} 2<4 \mathrm{WH}<\mathrm{BS} 3<\mathrm{BS} 1$ & BS3: $74 \%$ \\
\hline 3 & $\mathrm{EDCA}<4 \mathrm{WH}<\mathrm{BS} 1<\mathrm{BS} 2, \mathrm{BS} 3$ & N/A & BS3: $65 \%$ \\
\hline 4 & $\mathrm{BS} 1, \mathrm{BS} 2<4 \mathrm{WH}<\mathrm{EDCA}<\mathrm{BS} 3$ & N/A & BS3: $97 \%$ \\
\hline
\end{tabular}

Similarity Similarity to EDCA w/o Hidden Nodes w/o RTS/CTS, 4 WH EDCA with RTS/CTS, BS1 BusySiMOn v1, BS2 BusySiMOn v2, BS3 BusySiMOn v3

was done using the following metrics: overall network throughput (computed as the sum of all per-flow throughput values), fairness (measured with Jains fairness index), and similarity to a network without hidden nodes for the basic channel access, which was calculated using the following equation:

$$
\begin{aligned}
& \text { Similarity }= \\
& =\frac{O N T_{\text {BusySiMOn, network } w / \text { hidden nodes }}}{O N T_{\mathrm{EDCA}, \text { network } w / o \text { hidden nodes }}} \times 100 \% .
\end{aligned}
$$

The general conclusions regarding all of the tested networks are the following:

- BusySiMOn v3 performs the best in terms of the overall network throughput and fairness.

- The similarity of BusySiMOn v3 to EDCA without hidden nodes is within the range $65-100 \%$, which is very good because for other protocols the similarity factor is lower.

- If VO traffic is dominant (Configurations 1 and 3) BusySiMOn v1 and v2 are better than EDCA with and without RTS/CTS. This is because of the increased probability of successful wireless channel reservation during the preliminary reservation phase.

- In Configuration 3 for EDCA, nodes can hardly send any data. Such performance is unacceptable.
Additional detailed conclusions are presented next. For the three-node network (Fig. 4a):

- In Configuration 2 the fairness of BusySiMOn v2 is lower than for RTS/CTS. However, this is not very meaningful because for BusySiMOn v2 each node obtains higher throughput than for RTS/CTS.

For the four-node network (Fig. 4b):

- In Configuration 2 EDCA performs slightly better than BusySiMOn v1 in terms of the overall network throughput. However, BusySiMOn v1 assures much better fairness.

For the five-node network (Fig. 4c):

- BusySiMOn v1 is always better than EDCA and EDCA with RTS/CTS in terms of both the overall network throughput and fairness.

- In the first configuration RTS/CTS lowers the throughput of nodes in comparison to EDCA. On the other hand, it considerably improves fairness.

For the complex star topology network (Fig. 4d):

- In Configuration 2 EDCA performs better than $\mathrm{Bu}-$ sySiMOn v1 in terms of the overall network throughput. However, BusySiMOn v1 assures much better fairness. 
- In Configuration 4 EDCA performs better than BusySiMOn v1 because BK traffic has large $C W$ values and NO (transmitting VO traffic) is the only unhidden node. Therefore, in this configuration it is better if NO does not make any channel reservations and avoids unnecessary decrease of throughput.

To summarize the performance of the studied MAC protocols, it can be concluded that EDCA does not work properly in Configurations 1 and 3. This situation is slightly improved by the four-way handshake mechanism and even more improved by the BusySiMOn protocols. Additionally, even though the BusySiMOn protocols do not always improve the performance of EDCA in Configurations 2 and 4 , they perform satisfactorily in these configurations and, therefore, their overall performance is very adequate. This is in contrary to EDCA which has extremely low overall throughput in Configurations 1 and 3. Among the three BusySiMOn protocols BusySiMOn v3 performs most convincingly. Therefore, it is the best candidate for implementation in a real driver.

\subsubsection{Scenario 3: performance study under non-saturation}

When analyzing the performance of BusySiMOn under non-saturation the following set of outcomes is expected:

1. the throughput should be the highest,

2. the maximum frame delay of VO traffic should be less than $150 \mathrm{~ms}$,

3. the performance of the protocol should be the best when VO is the dominant traffic in the network (Configurations 1 and 3), slightly worse performance for BK traffic can be accepted (Configurations 2 and 4 ).

7.1.4.1 Results So far, it has been shown that the overall performance of BusySiMOn (especially BusySiMOn v3) is better than the performance of EDCA and the four-way handshake mechanism in terms of the overall network throughput and fairness when VO is the dominant traffic in the network. However, for delay-sensitive traffic it is the maximum frame delay which is the most important constraint. We assume that a VO service can tolerate a maximum frame delay of $150 \mathrm{~ms}$. Frames with greater delay are dropped. Therefore, the maximum values of the perflow offered load were found under which the wireless network was not yet saturated. This was done separately for BusySiMOn v3 and the four-way handshake for Configuration $1(45 \mathrm{~KB} / \mathrm{s}$ and $53.75 \mathrm{~KB} / \mathrm{s}$, respectively) and Configuration $3(56.88 \mathrm{~KB} / \mathrm{s}$ and $63.75 \mathrm{~KB} / \mathrm{s}$, respectively). Then, the two protocols were compared together and, additionally, with BusySiMOn v1 and v2. The comparison was done with regard to the overall throughput and the maximum frame delay obtained for the acquired values of the network load. This scenario was executed for the most complex network illustrated in Fig. 4d. Table $7^{6}$ contains the obtained results.

The general conclusions are the following:

- Only BusySiMOn v3 assures fulfillment of delay constraints for VO traffic.

- The maximum throughput of the four-way handshake under the delay constraint is worse than the throughput of each BusySiMOn protocol.

- The maximum delay of BK traffic is always lower for the BusySiMOn protocols than for the four-way handshake mechanism.

- The fairness of the BusySiMOn protocols is always higher than the fairness of the four-way handshake mechanism.

To summarize, in each analyzed configuration the new mechanisms perform better than EDCA with RTS/ CTS not only in terms of the maximum frame delay but also in terms of fairness and overall network throughput. This means that with the use of the BusySiMOn protocols delaysensitive traffic is provided with a better level of QoS than with the use of the four-way handshake mechanism.

\subsection{Scenario 4: Four ACs per node-performance study under saturation}

In this section the impact of virtual collisions on the performance of BusySiMOn $v 1^{7}$ and the four-way handshake mechanism is investigated. The two protocols were evaluated in the network illustrated in Fig. 5, which consists of two collision domains. N0 is the only unhidden node. The number of nodes hidden from each other was increased from 10 to 40 (i.e., there were from 5 to 20 nodes in each domain). Hidden nodes were simultaneously transmitting data belonging to all four ACs to N0. Node N0 did not transmit any data.

The results are presented in Fig. 6. For clarity of presentation the figure illustrates throughput only for two ACs (solid lines). The VO and BK ACs were chosen as the most opposite. Additionally, the figure illustrates the overall throughput per collision domain (dashed lines), which is the sum of throughput of all ACs.

The conclusions are the following:

\footnotetext{
${ }^{6}$ Table 7 does not contain the results for BusySiMOn v2, because in Configuration 1 they were the same as for BusySiMOn v1 and in Configuration 3 they were the same as for BusySiMOn v3.

${ }^{7}$ In this scenario the performance of BusySiMIOn v3 is similar to the performance of BusySiMOn v1 because only hidden nodes transmit data.
} 
Table 7 Results for complex star topology (Fig. 4d) for non-saturation

\begin{tabular}{llllllllllllll}
\hline PFOL & $\mathrm{C}$ & $\begin{array}{l}\text { ONT } \\
(\mathrm{RTS})\end{array}$ & $\begin{array}{l}\mathrm{F} \\
(\mathrm{RTS})\end{array}$ & $\begin{array}{l}\mathrm{AFD}^{1} \\
(\mathrm{RTS})\end{array}$ & $\begin{array}{l}\mathrm{AFD}^{2} \\
(\mathrm{RTS})\end{array}$ & $\begin{array}{l}\text { ONT } \\
(\mathrm{BS} 1)\end{array}$ & $\begin{array}{l}\mathrm{F} \\
(\mathrm{BS} 1)\end{array}$ & $\begin{array}{l}\mathrm{AFD}^{1} \\
(\mathrm{BS} 1)\end{array}$ & $\begin{array}{l}\mathrm{AFD}^{2} \\
(\mathrm{BS} 1)\end{array}$ & $\begin{array}{l}\mathrm{ONT}^{2} \\
(\mathrm{BS3})\end{array}$ & $\begin{array}{l}\mathrm{F}^{2} \\
(\mathrm{BS3})\end{array}$ & $\begin{array}{l}\mathrm{AFD}^{1} \\
(\mathrm{BS3})\end{array}$ & $\begin{array}{l}\mathrm{AFD}^{2} \\
(\mathrm{BS3})\end{array}$ \\
\hline 45 & 1 & 175.64 & 0.52 & 9.9 & $65-150$ & 351.43 & 0.89 & 3.2 & 3.5 & 351.13 & 0.89 & 2.27 & 2.95 \\
53.75 & 1 & 185.10 & 0.46 & 13.7 & 11,000 & 349.54 & 0.88 & 5,400 & 11,000 & 420.30 & 0.89 & 3.8 & 8 \\
56.88 & 3 & 108.52 & N/A & 165,000 & $88-113$ & 435.90 & N/A & 4,750 & 4.3 & 443.94 & N/A & 10.3 & 3.5 \\
63.75 & 3 & 94.29 & N/A & 167,500 & 8,200 & 428.67 & N/A & 8,860 & 6.5 & 462.10 & N/A & 5,490 & 5.1 \\
\hline
\end{tabular}

$P F O L$ Per-Flow Offered Load [KB/s], $C$ configuration, $O N T$ overall network throughput $[\mathrm{KB} / \mathrm{s}], F$ fairness, $R T S$ EDCA with RTS/CTS, $A F D^{1}$ average frame delay for N0 [ms], $A F D^{2}$ average frame delay for other nodes [ms], BS1 BusySiMOn v1, BS3 BusySiMOn v3

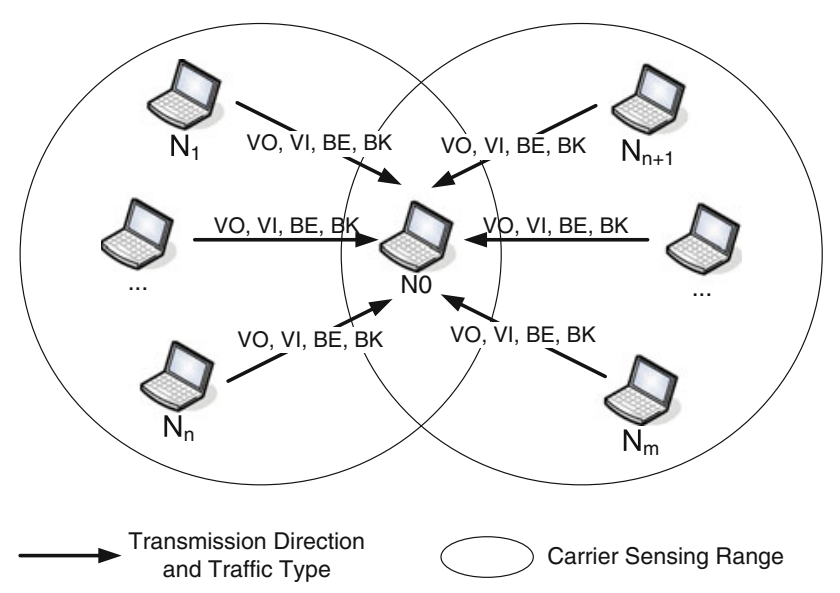

Fig. 5 Simulated network with four ACs per node

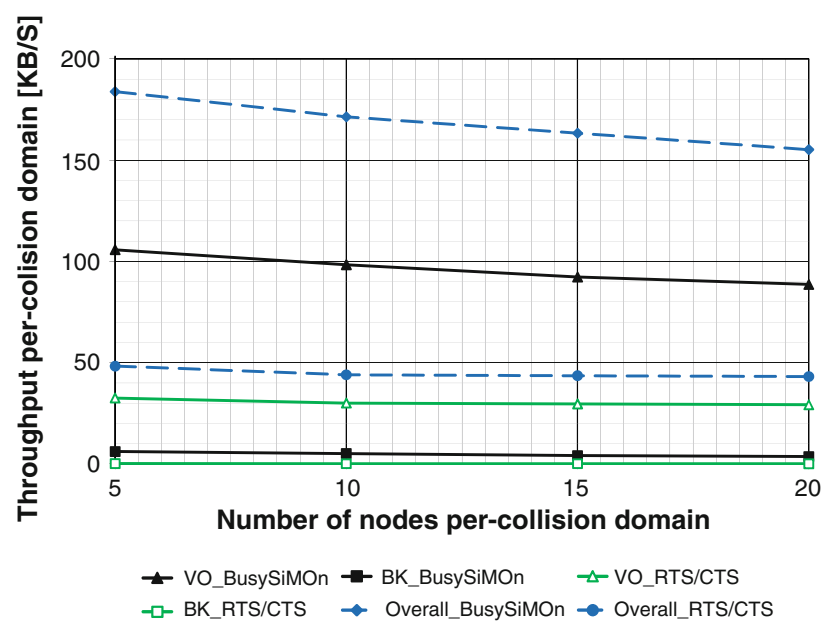

Fig. 6 Results for the network with four ACs per node

- Both the four-way handshake mechanism and BusySiMOn provide proper traffic prioritization.

- BusySiMOn assures over 3.5 times higher network utilization than the four-way handshake mechanism.

- The increase of the number of nodes in each domain does not meaningfully impact the per-AC throughput.
For EDCA without RTS/CTS the hidden nodes could not successfully transmit any data due to a large number of collisions. Therefore, these unsatisfying results are not presented here.

\section{Estimation of energy consumption}

In BusySiMOn the transmission of additional busy tone signals (Busy 1 and Busy 2) consumes additional energy to complete the reservation procedure, compared to the fourway handshake mechanism. In this section the power consumption overhead is analyzed.

\subsection{Total energy consumption}

The total energy consumed by a WLAN (Wireless Local Area Network) card includes (i) energy consumed when transmitting frames (e.g. DATA, RTS, CTS) or busy tones, (ii) energy consumed in the process of receiving frames, and (iii) energy consumed when the device is idle. It is obvious that more power is consumed when performing a data transfer than when receiving frames, however, power consumption of an idle device is also not negligible. In an idle state the WLAN device senses the wireless channel to detect its state and to detect the start of a new frame transmission. This consumes significant amount of energy.

Table 8 shows power consumed by several popular WLAN chipsets in each of the three states: transmission, reception, and idle [33]. As anticipated, the older cards (e.g., WaveLAN, Intersil PRISM I) manufactured with older technology consume more energy than modern IEEE 802.11 combo cards.

\subsection{Energy consumption overhead}

We have compared power consumption of BusySiMOn v1 (which is always using the additional Busy 1 and Busy 2 signals) and EDCA with the four-way handshake mechanism enabled in a network without hidden nodes. In the comparison we assumed IEEE $802.11 \mathrm{~b}$ as the default PHY layer. The wireless channel introduced no errors. The 
Table 8 Power consumption rates of different vendor 802.11 WLAN cards for transmission (Tx), reception (Rx), and idle states

\begin{tabular}{llllllll}
\hline Device & WaveLAN & $\begin{array}{l}\text { Atheros } \\
\text { AR5001X }\end{array}$ & $\begin{array}{l}\text { Intel Pro } \\
\text { Wireless 2100 }\end{array}$ & $\begin{array}{l}\text { Intersil } \\
\text { PRISM I }\end{array}$ & $\begin{array}{l}\text { Cisco } \\
\text { Aironet 350 }\end{array}$ & $\begin{array}{l}\text { Intel Pro Wireless } \\
\text { 3945ABG }\end{array}$ & $\begin{array}{l}\text { Cisco aironet AIR- } \\
\text { CB21AG }\end{array}$ \\
\hline Supported & $802.11 /$ & $802.11 /$ & $802.11 / 802.11 \mathrm{~b}$ & $802.11 / 802.11 \mathrm{~b}$ & $802.11 /$ & $802.11 \mathrm{abg}$ & $802.11 \mathrm{abg}$ \\
PHY & $802.11 \mathrm{~b}$ & $802.11 \mathrm{~b}$ & & & $802.11 \mathrm{~b}$ & & \\
Tx [W] & 1.65 & 1.35 & 1.91 & 2.50 & 1.75 & 1.80 & 1.80 \\
Rx [W] & 1.40 & 1.02 & 1.39 & 0.90 & 1.25 & 1.40 & 1.05 \\
Idle [W] & 1.15 & 0.89 & 0.29 & 0.11 & 0.19 & 0.15 & 0.67 \\
\hline
\end{tabular}

DATA rate was set to $11 \mathrm{Mb} / \mathrm{s}$, and the basic rate was set to $1 \mathrm{Mb} / \mathrm{s}$. The size of DATA frames was set to $1000 \mathrm{~B}$. The network consisted of two nodes: one was receiving and the second was transmitting. Two different configurations were considered:

- Configuration 1: transmission of Vo traffic, saturation conditions.

- Configuration 2: transmission of BK traffic, saturation conditions.

Let the time period for which the WLAN card is in transition, reception, and idle mode be $T_{t}, T_{r}$, and $T_{i}$, respectively. Additionally, let the power consumed in these states be $P_{t}, P_{r}$, and $P_{i}$, respectively. Then, the energy consumption can be defined as $P_{\text {cons }}=P_{t} T_{t}+P_{r} T_{r}+P_{i}$ $T_{i}$. Now we can derive the equations for a single transmission period of BusySiMOn and EDCA with the four way-handshake mechanism enabled. The transmitting and receiving nodes are marked with subscripts $\mathrm{Tx}$ and $\mathrm{Rx}$, respectively.

$$
\begin{aligned}
& P_{\text {cons }}\left[\text { BusySiMOn }_{T x}\right]= \\
& =P_{t} \times\left(T^{\text {Busy } 1}+T^{R T S}+T^{D A T A}\right)+ \\
& +P_{r} \times\left(T^{\text {Busy } 2}+T^{C T S}+T^{A C K}\right)+ \\
& +P_{i} \times(A I F S+\text { Backoff }+5 \times S I F S) \\
& P_{\text {cons }}\left[\text { BusySiMOn }_{R x}\right]= \\
& =P_{t} \times\left(T^{\text {Busy } 2}+T^{C T S}+T^{A C K}\right)+ \\
& +P_{r} \times\left(T^{\text {Busy } 1}+T^{R T S}+T^{D A T A}\right)+ \\
& +P_{i} \times(\text { AIFS }+ \text { Backoff }+5 \times S I F S) \\
& P_{\text {cons }}\left[E D C A_{T x}\right]= \\
& =P_{t} \times\left(T^{R T S}+T^{D A T A}\right)+ \\
& +P_{r} \times\left(T^{C T S}+T^{A C K}\right)+ \\
& +P_{i} \times(\text { AIFS }+ \text { Backoff }+3 \times \text { SIFS }) \\
& P_{\text {cons }}\left[E D C A_{R x}\right]= \\
& =P_{t} \times\left(T^{C T S}+T^{A C K}\right)+ \\
& +P_{r} \times\left(T^{R T S}+T^{D A T A}\right)+ \\
& +P_{i} \times(\text { AIFS }+ \text { Backoff }+3 \times \text { SIFS })
\end{aligned}
$$

Based on the presented equations it is possible to calculate the amount of energy required for a single DATA transmission by different WLAN cards using either BusySiMOn or EDCA with the four-way handshake mechanism enabled. Figures 7 and 8 present the consumed power defined in watts required for a single DATA transmission for Configurations 1 and 2, respectively.

The obtained results show that the difference in energy consumption during a single DATA frame transmission between BusySiMOn and EDCA can reach up to $6 \%$. This is caused by the additional signaling overhead introduced by the BusySiMOn protocol. The difference is smaller for a transmission state than for a reception state. Moreover, it is almost independent from the type of the IEEE 802.11 WLAN card. Finally, all WLAN cards consume more energy in Configuration 2 (transmitting BK traffic) than in Configuration 1 (transmitting Vo traffic). This is because of the lager Backoff periods of the node transmitting $\mathrm{BK}$ traffic compared to the Backoff periods of the node transmitting Vo traffic.

\section{Comparison with other protocols}

In this section we explain the differences in operation of BusySiMOn and other busy tone-based protocols (PUMA and Black Burst). Additionally, we clarify why we compared BusySiMOn only with the four-way handshake mechanism (operating together with EDCA) and not with other QoS-aware MAC protocols (cf. Table 1).

\subsection{Busy tone-based protocols}

PUMA and Black Burst both operate similarly. In Black Burst, nodes sending real-time traffic use pulses of energy, which are called Black Bursts (BB), to contend for medium access. The length of these pulses is proportional to the time the nodes had to wait for the channel to become idle. This delay is measured from the first attempt to access the channel by a node until its transmission starts. After transmitting its $\mathrm{BB}$, the node waits for a specified time interval to see if any other node is transmitting a longer $\mathrm{BB}$. If the channel is perceived idle after this interval, then the node can immediately transmit its real-time frame. 
Fig. 7 Power consumption of 802.11 WLAN cards required for a single DATA frame transmission using BusySiMOn and EDCA with the four-way handshake mechanism enabled in Configuration 1
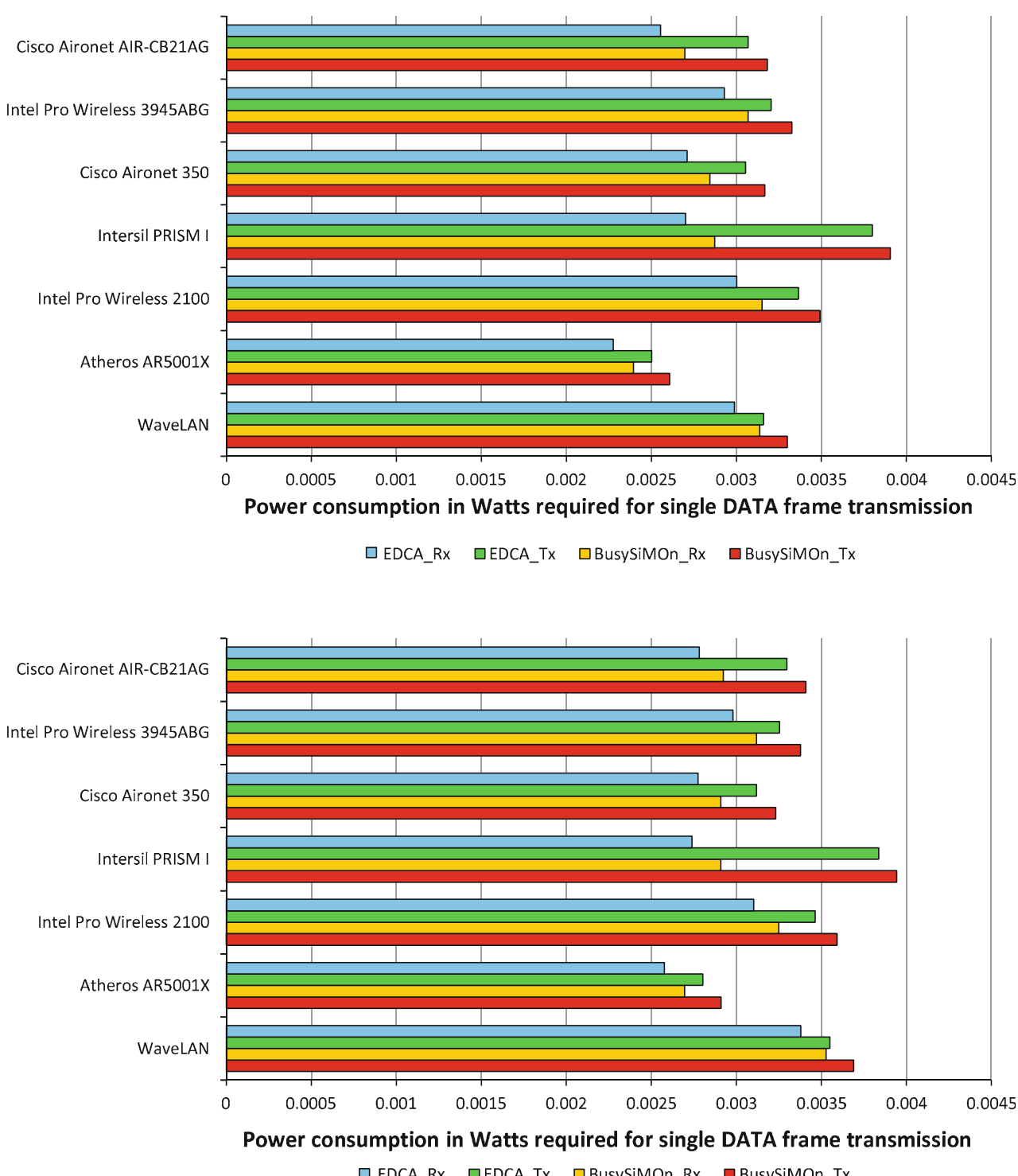

$\square$ EDCA_Rx $\square$ EDCA_Tx $\square$ BusySiMOn_Rx aBusySiMOn_Tx
Fig. 8 Power consumption of 802.11 WLAN cards required for a single DATA frame transmission using BusySiMOn and EDCA with the four-way handshake mechanism enabled in Configuration 2

\subsection{QoS-aware MAC protocols}

Otherwise, it waits for the next channel access cycle and time frames start its transmission simultaneously (after PIFS) and send a JAM signal. The JAM signal consists of pulses of energy and has the length of one slot. This signal informs all other nodes (especially nodes sending non realtime frames) that in their neighborhood a real-time transmission will begin.

In BusySiMOn the situation is slightly different because not only the sender but also the destination node transmits the jamming signals (Busy 1 and Busy 2, respectively). Additionally, BusySiMOn uses these signals to, most of all, alleviate the hidden node problem and not for traffic prioritization (cf. BusySiMOn v3). Traffic prioritization in BusySiMOn is realized through EDCA compatibility (in particular, the support of AC-dependent AIFSN, CW and TXOPLimit values).
The state of the art (Table 1) there are three types of protocols which support QoS:

- Busy tone-based (Black Burst, PUMA): these protocols do not support the EDCA traffic categories defined by the IEEE 802.11 standard. Therefore, a comparison of BusySiMOn with this group of protocols would not be complete.

- $\quad$ RTS/CTS-based (AA, M-VRMA, DRCE, EDCA/RR): not all of these protocols are backward compatible with EDCA. But most of all, their operation is based on the RTS/CTS frames (sometimes modified) and, therefore, they perform similarly to the four-way handshake mechanism in medium access. The Busy 1 and Busy 2 signals proposed by BusySiMOn are shorter than the 
signaling frames introduced by this group of protocols and, therefore, the probability of successful reservation of the wireless channel is higher for BusySiMOn. This was shown for the four-way handshake mechanism in our paper.

- Binary countdown-based (RICK): the medium access method used by this group of protocols is not backward compatible with the IEEE 802.11 standard. Additionally, they do not support the access categories defined by EDCA.

Based on the above explanation, it appears reasonable that the performed comparison of BusySiMOn with the four-way handshake protocol (operating together with EDCA) is the most accurate and adequate.

\section{Conclusions}

This article has presented a new method of preliminary reservation of the wireless channel for IEEE 802.11 ad-hoc networks with hidden nodes. The simulation results have demonstrated that the currently used four-way handshake mechanism is inefficient, especially for high priority flows transmitted by hidden nodes. It has been shown that all three versions of the BusySiMOn protocol can improve network performance. The new solutions have been shown to outperform the four-way handshake mechanism not only in the case of physical collisions but also in the case of virtual ones. Furthermore, since the operation of BusySiMOn v3 is the most promising it is the best candidate for implementation in a real driver.

The key advantage of all three versions of BusySiMOn is the minimized risk of collisions of signaling data during the preliminary wireless channel reservation. This is of great importance especially for high priority traffic because it results in increased channel efficiency, reduced maximum frame delay and improved fairness among the nodes. Additionally, the combination of the preliminary reservation procedure with the RTS/CTS exchange and the unchanged values of the EDCA access parameters assures compatibility with mechanisms implemented in current wireless devices and makes it an ideal candidate for future, enhanced implementation.

Acknowledgments This work has been supported by the European Community's Seventh Framework Programme (FP7-SEC-2011-1) under grant agreement n. 285320 (PROACTIVE project).

Open Access This article is distributed under the terms of the Creative Commons Attribution License which permits any use, distribution, and reproduction in any medium, provided the original author(s) and the source are credited.

\section{Appendix: A probability of successful channel reservation by hidden nodes}

In this appendix we explain how to calculate the lower bound of the probabilities of successful channel reservation by hidden nodes when the four-way handshake mechanism $\left(p_{s}^{H, R T S}\right)$ and BusySiMOn $\left(p_{s}^{H, B u s y 1}\right)$ are used, respectively. Similarly as in Sect. 4, we consider the first scenario illustrated in Fig. 2. Additionally, for clarity of presentation, we consider the OFDM PHY with $20 \mu$ s of PLCP overhead, an STP of $9 \mu \mathrm{s}$, and a SIFS of $16 \mu \mathrm{s}$.

Four-way handshake

First, we consider a situation in which one of the hidden nodes transmits an RTS frame to the middle node. The assumed OFDM PHY determines the number of STPs required to transmit the RTS frame (together with the PLCP overhead), which is equal to 5.19. After the RTS frame is correctly received, the middle node listens to the wireless channel for a SIFS period and then, if the channel is idle, it transmits a CTS frame. Therefore, we calculate the natural number of STPs required to correctly complete the described procedure $\left(T_{R T S}\right)$ :

$T_{R T S}=\lceil R T S+S I F S\rceil$,

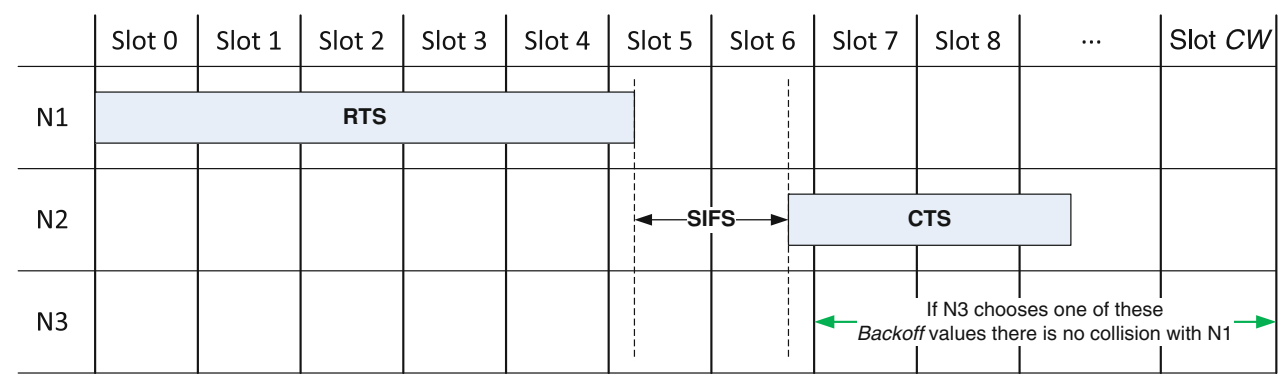

Fig. 9 Successful channel reservation by a hidden node for the OFDM PHY with $20 \mu$ s of PLCP overhead. RTS includes the PLCP overhead 
where RTS and SIFS are the numbers of STPs required to transmit the RTS frame and wait for the SIFS period, respectively. In Fig. 9 we present an exemplary successful channel reservation by one of the hidden nodes.

For the assumed PHY, $T_{R T S}$ is equal to seven, therefore, if we additionally assume a Backoff in which $C W+1$ is equal to eight, we can calculate the probability of successful channel reservation by either of the hidden nodes $p_{s}^{H}$. It is equal to the probability that one of the hidden nodes transmits without any Backoff (i.e., in slot 0 ) and the other one chooses seven Backoff slots (i.e, it can transmit in slot 7 if the medium is idle). In all other cases $p_{s}^{H}$ equals zero. We calculate this as

$p_{s}^{H, C W=7}=\left(\begin{array}{l}2 \\ 1\end{array}\right) \frac{1}{C W+1} \times \frac{C W+1-T_{R T S}}{C W+1}$.

Similarly, for $C W$ equal to 15 , the successful channel reservation by either of the hidden nodes is possible if one of the hidden nodes chooses a Backoff value of 0 and the other hidden node chooses $7,8, \ldots$, or 15 , or if one of the hidden nodes chooses a Backoff value of 1 and the other hidden node chooses $8,9 \ldots$, or 15 , etc. We calculate this as

$$
\begin{aligned}
p_{s}^{H, C W=15} & =\left(\begin{array}{l}
2 \\
1
\end{array}\right)\left(\frac{1}{C W+1} \frac{C W+1-T_{R T S}}{C W+1}+\right. \\
& \left.+\frac{1}{C W+1} \frac{C W-T_{R T S}}{C W+1}+\ldots+\frac{1}{C W+1} \frac{1}{C W+1}\right)= \\
& =\frac{\left(C W-T_{R T S}+1\right)\left(C W-T_{R T S}+2\right)}{(C W+1)^{2}} .
\end{aligned}
$$

For larger $C W s$ general equations for $p_{s}^{H}$ are the same as Eq. 2.

Taking into account different PHYs (cf. Table 3) it may occur that the number of STPs required for $T_{R T S}$ is larger than the assumed $C W$ (i.e., $\alpha=C W-T_{R T S}<0$ ). If this case $p_{s}^{H}$ is equal to zero. Therefore, from this observation as well as (1) and (2) we get the general equation for the probability of the successful channel reservation by either of the hidden nodes for the four-way handshake mechanism $\left(p_{s}^{H, R T S}\right)$ :

$p_{s}^{H, R T S}=\left\{\begin{array}{cc}0, & \text { if } \alpha<0 \\ \frac{(\alpha+1)(\alpha+2)}{(C W+1)^{2}}, & \text { if } \alpha \geq 0\end{array}\right.$.

\section{BusySiMOn}

For BusySiMOn, the calculation of the lower bound of the probability of successful channel reservation by the hidden nodes $\left(p_{s}^{H, B u s y}{ }^{1}\right)$ is the same as for the four-way handshake. The only difference is that instead of the RTS frame we take into account the Busy 1 signal:
$p_{s}^{H, B u s y 1}=\left\{\begin{array}{cl}0, & \text { if } \beta<0 \\ \frac{(\beta+1)(\beta+2)}{(C W+1)^{2}}, & \text { if } \beta \geq 0\end{array}\right.$,

where $\beta=C W-T_{\text {Busy } 1}$.

\section{References}

1. Bharghavan, V., Demers, A. J., Shenker, S., \& Zhang, L. (1994). MACAW: A media access protocol for wireless LAN's. In Proceedings of SIGCOMM.

2. Chang, J.-J., Liao, W., \& Hou, T.-C. (2009). Reservation-based directional medium access control (RDMAC) protocol for multihop wireless networks with directional antennas. In Proceedings of IEEE international conference on communications.

3. Choudhury, R. R., Yang, X., Ramanathan, R., \& Vaidya N. H. (2002). Using Directional Antennas for Medium Access Control in Ad Hoc Networks. In Proceedings of the ACM annual international conference on mobile computing and networking.

4. Fullmer, C. L., \& Garcia-Luna-Aceves, J. J. (1995). Floor acquisition multiple access (FAMA) for packet-radio networks. In Proceedings of ACM SIGCOMM.

5. Haas, Z. J., Deng, J. (2002). Dual busy tone multiple access (DBTMA): a multiple access control scheme for ad hoc networks. IEEE Transactions on Communications 50(6), 975-984.

6. Hamidian, A., \& Korner U.(2006). Providing QoS guarantees by enhancing IEEE 802.11e through EDCA with resource reservation. In Proceedings of workshop on wireless and mobility.

7. Huang, R., Zhai, H., Zhang, C., \& Fang Y. (2008). SAM-MAC: an efficient channel assignment scheme for multi-channel ad hoc networks. Computer Networks, Elsevier, 52, 1634-1646.

8. IEEE 802.11, (2007, June), Wireless LAN medium access control (MAC) and Physical Layer (PHY) Specifications, IEEE Inc., USA: New York

9. Jain, R., Chiu, D., \& Hawe, W.(1984). A quantitative measure of fairness and discrimination for resource allocation in shared computer systems, DEC research report TR-301.

10. Jung, E. S., \& Vaidya, N. H. (2002). A power control MAC protocol for ad hoc networks. In Proceedings of the ACM annual international conference on mobile computing and networking.

11. Korakis, T., Jakllari, G., \& Tassiulas, L. (2007). CDR-MAC: A protocol for full exploitation of directional antennas in ad hoc wireless networks. IEEE Transactions On Mobile Computing, 6, (12).

12. Kosek, K., Natkaniec, M., Vollero, L., \& Pach, A. R. (2008). Performance analysis of 802.11e networks with hidden nodes in a star topology. In Proceedings of IEEE consumer communications and networking conference.

13. Kosek, K., Natkaniec, M., \& Vollero, L. (2008). Thorough analysis of 802.11e star topology scenarios in the presence of hidden nodes. In Proceedings of IFIP networking.

14. Kosek-Szott, K., Natkaniec, M., \& Pach, A. R. (2011). A simple but accurate throughput model for IEEE 802.11 EDCA in saturation and non-saturation conditions. Computer Networks, Elsevier, 55(3), 622-635.

15. Kosek-Szott, K., Natkaniec, M., \& Pach, A. R. (2010). BusySiMOn: a new protocol for IEEE 802.11 EDCA-based ad-hoc networks with hidden nodes. In Proceedings of IEEE global communications conference.

16. Lai, W. K., Tseng, K.-S., \& Chen, J.-C. (2009). MARS: A multiple access scheme with sender driven and reception first for smart antenna in ad hoc networks. Wireless Communications and Mobile Computing, 9(2). 
17. Monks, J., Bharghavan, V., \& Hwu, W. (2001). A power controlled multiple access protocol for wireless packet networks. In Proceedings of IEEE international conference on computer communications.

18. Moon, Y., \& Syrotiuk, V. R. (2009) Acooperative CDMA-based multi-channel MAC protocol for mobile ad hoc networks. Elsevier, Computer Communications, 32(17), 1810-1819.

19. Natkaniec, M., \& Pach, A. R. (2002). PUMA: A new channel access protocol for wireless LANs. In Proceedings of wireless personal multimedia communications.

20. Singh, R. P., \& Lobiyal, D. K. (2009). Performance modeling of slotted MACA-BI MAC protocol for mobile ad hoc networks. In Proceedings of ACM international conference on computers and information systems.

21. Sobrinho, J. L., \& Krishnakumar, A. S. (1996). Real-time traffic over the IEEE 802.11 medium access control layer. Bell Labs Technical Journal 1(2), 172-187.

22. Takatsuka, Y., Takata, M., Bandai, M., \& Watanabe, T. (2008). A MAC protocol for directional hidden terminal and minor lobe problems. In Proceedings of IEEE wireless telecommunications symposium.

23. Varvarigos, E. A., Vasileios, G., \& Nikolaos, K. (2009). The slow start power controlled MAC protocol for mobile ad hoc networks and its performance analysis. Elsevier, Ad Hoc Networks, 7, 1136-1149.

24. Yeh, C.-H., Zhou, H., Ho, P.-H., \& Mouftah, H. T.(2003). A variable-radius multichannel MAC protocol for high-throughput low-power heterogeneous ad hoc networking. In Proceedings of IEEE global communications conference.

25. Yeh, C.-H. (2003). The advance access mechanism for differentiated service, power control, and radio efficiency in ad hoc MAC protocols. In Proceedings of IEEE vehicular technology conference.

26. Yeh, C.-H. (2004). A new scheme for effective MAC-layer DiffServ supports in mobile ad hoc networks and multihop wireless LANs. In Proceedings of IEEE vehicular technology conference.

27. Yeh, C.-H. (2004). A collision-controlled MAC protocol for mobile ad hoc networks and multihop wireless LANs. In Proceedings of IEEE global communications conference.

28. You, T., Yeh, C.-H., \& Hassanein, H. (2005). DRCE: a high throughput QoS MAC protocol for wireless ad hoc networks. In Proceedings of IEEE symposium on computers and communications.

29. Li, F.Y., Kristensen, A., \& Engelstad, P.E. (2006). Passive and active hidden terminal detection in 802.11-based ad hoc networks. In Proceedings of IEEE international conference on computer communications.

30. Li, F., Kristensen, A., \& Engelstad, P. (2006). Hidden terminal detection in 802.11-based wireless ad hoc networks. In Proceedings of the 15th IST mobile and wireless communication summit.

31. Mostefa, F. Z., Mekkakia, M. Z., \& Khelifa, S. (2011). Techniques of detection of the hidden node in wireless ad hoc network. In Proceedings of the World Congress on Engineering.

32. Myles, A. F., Lam, A. C. K., \& Goodall, D. S. (2007). Detecting, reporting and mitigating hidden nodes in a wireless data network. Patent number: 7184407. Issue date: February 27.
33. Chiaravalloti, S., Idzikowski, F., \& Budzisz, L. (2011). Power consumption of WLAN network elements. TKN technical report TKN-11-002, August 2011.

34. Natkaniec, M., \& Pach, A. R. (2000). A performance analysis of IEEE 802.11 networks in the presence of hidden stations. In Proceedings of the IFIP TC6, WG6.8 5th workshop on personal wireless communication (PWC2000) (pp. 157- 168). Gdansk: Kluwer.

\section{Author Biographies}

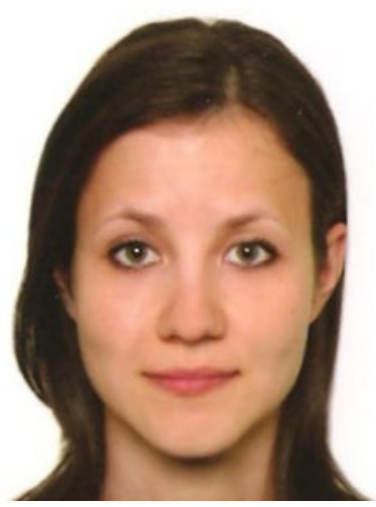

Katarzyna Kosek-Szott received her M.Sc. and Ph.D degrees in telecommunications (both with honours) from the AGH University of Science and Technology, Krakow, Poland in 2006 and 2011, respectively. Currently she is working as an assistant professor at the Department of Telecommunications, AGH University of Science and Technology.Her general research interests are focused on wireless networking. The major topics include wireless LANs (especially ad-hoc networks) and quality of service provisioning within these networks. She is a reviewer for international journals and conferences. She has been involved in several European projects: DAIDALOS II, CONTENT, CARMEN, FLAVIA, PROACTIVE as well as grants supported by the Polish Ministry of Science and Higher Education. She has also co-authored a number of research papers.

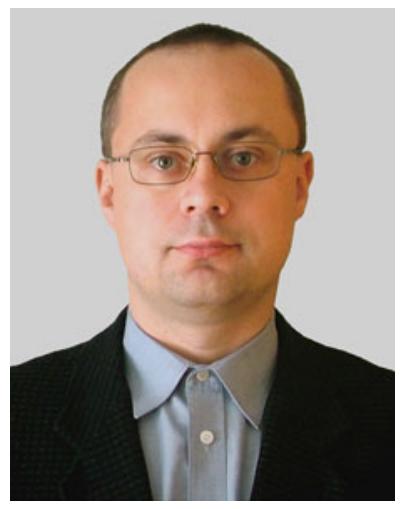

Marek Natkaniec received the M.Sc. and Ph.D. degrees in telecommunications from the Faculty of Electrical Engineering, AGH University of Science and Technology, Cracow, Poland in 1997 and 2002, respectively. In 1997 he joined AGH University of Science and Technology. Now, he works as an assistant professor at the Department of Telecommunications. His general research interests are in wireless networks. Particular topics include wireless LANs, designing of protocols, modeling and performance evaluation of communication networks, quality of service, and cooperation of networks. $\mathrm{He}$ is a reviewer to international journals and conferences. He has/had actively participated in European projects: MOCOMTEL, PROACCESS, DAIDALOS I, DAIDALOS II, CONTENT, CARMEN, MEDUSA, HECTOR, FLAVIA, PROACTIVE as well as grants 
supported by the Ministry of Science and Higher Education. He is involved in standardization activities for ETSI. He serves as an expert and a consultant to telecom operators in the area of wireless networks. Marek Natkaniec co-authored five books and over 100 research papers. $\mathrm{He}$ is a senior member of IEEE.

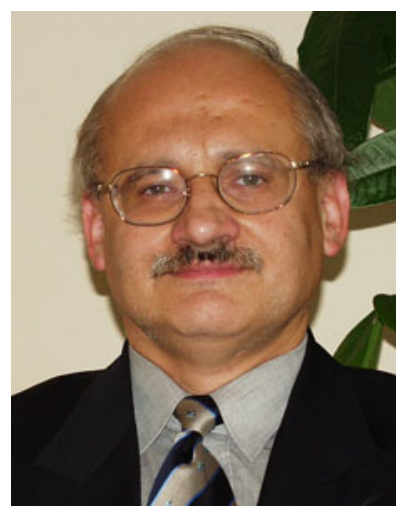

Andrzej R. Pach received the M.S. degree in electrical engineering and the Ph.D. degree in telecommunications from the University of Science and Technology, Krakow, Poland, in 1976 and 1979, respectively, and the Ph.D.Hab. in telecommunications and computer networks from the Warsaw University of Technology in 1989. In 1979, he joined the Department of Telecommunications at the University of Science and Technology, where he is currently a Professor and Chair. He spent his sabbatical leaves at CNET, France and University of Catania, Italy. He is the VicePresident of the Foundation for Progress in Telecommunications and serves as a chairman of the IEEE Communications Society Chapter. He has been a consultant to governmental institutions and telecom operators in modern telecommunication networks. His research interests include design and performance evaluation of broadband networks, especially quality of service and network performance of access networks and wireless LANs. He has/had actively participated in COST, Eureka Celtic, and 4th, 5th, 6th, 7th FP European programs (ACTS, ESPRIT, IST, ICT, and Security). He co-authored more than 250 publications including 6 books. He served as a technical editor to IEEE Communications Magazine and is an editor-in-chief to Digital Communications-Technologies and Services. He has also been appointed as an expert in Information and Communications Technologies by the European Commission. 